MILITARY TECHNICAL COLLEGE
CAIRO - EGYPT

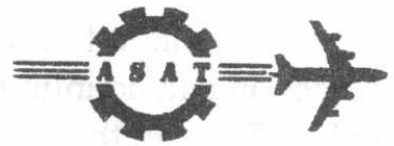

$7^{\text {th }}$ INTERNATIONAL CONF. ON AEROSPACE SCIENCES \& AVIATION TECHNOLOGY

\title{
Frequency Domain Parametric Identification of a Flexible Manipulator Model for Structural Control Applications
}

\author{
${ }^{*}$ A. E. Shawky ${ }^{* *}$ A. S. ABDEL-MOHSEN
}

\begin{abstract}
The problem of modeling a single-link flexible manipulator having a revolute joint, is rather complex. It involves complex interaction and coupling between the desired rigid body motion and the undesired flexible motion. Analysis is performed on a representative case study of a Pinned-Free Euler-Bernoulli beam with noncollocated sensors. Numerical method for accurate computation of the transfer function poles and zeros is proposed. Experimental investigations that have been carried out on a real laboratory model of a single link flexible manipulator is reported. This model consists of a prismatic beam free at one end and attached to a DC motor at the other end. Using a dual channel Dynamic Signal Analyzer DSA, the manipulator has been excited by a harmonic torque and the structural displacement at several locations have been measured using vibration accelerometer. The DSA curve fitting capability are used to determine the best fit estimate of the manipulator's experimental transfer function poles and
zeros.
\end{abstract}

\section{KEY WORDS}

Modeling, Identification, Control, Flexible manipulator.

\section{INTRODUCTION.}

One of the major limitations of current industrial robots is their low payload/weight ratio. Excessive arm weight not only limit the speed at which robots can displace from one point to another but also increases the energy requirements and the size of actuators. Manipulators made with lighter materials are flexible and cannot be controlled accurately with the jointoriented classical controllers used in most industrial robots. *M.Sc., Research Engineer, R\&D center for Air Force, Cairo, Egypt. ** Prof. \& Head of the
Department of the Aeronautics, M.T.C, Cairo, Egypt. 
The problem of modeling and control of a single-link flexible manipulator, in general is rather complex. It involves complex interaction and coupling between the desired rigid body motion and the undesired flexible motion $[2,4,5,9]$. In this work, however, only structural vibrational behavior of the manipulator will be considered, i.e. no large rigid rotation is considered. This case corresponds to the practical situation at the end of a rest-to-rest rotational maneuver of the flexible manipulator shown in Fig. 1. The structural behavior of the flexible manipulator, here, is considered to be that of an Euler-Bernoulli beam pinned at the joint hinge axis, and free at the other end. The structural transfer function of this manipulator has been derived on basis of the product expansion method [6,7]. Expressions for the transfer functions poles and zeros have been obtained. For the numerical computation of transfer function poles and zeros, a computer program based on Matlab Package has been prepared. Synthesized frequency response curves are obtained based on the computed poles and zeros.

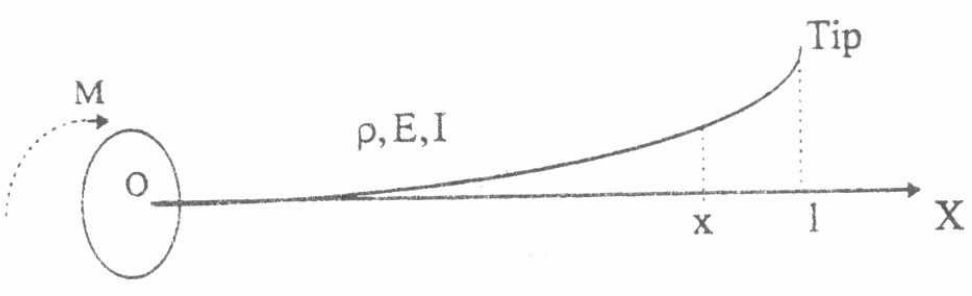

Fig. 1 Pinned-Free beam model

Experimental investigations that have been carried out on a real laboratory model of a singlelink flexible manipulator is reported. This model consists of a prismatic beam free at one end and attached to a DC motor at the other end. Using a dual channel Dynamic Signal Analyzer DSA, the manipulator has been excited by a harmonic torque and the structural displacement at several locations have been measured using vibration accelerometers, The DSA process the torque and displacement signals and yields the frequency response function. The DSA curve fitting capability are used to determine the best fit estimate of the manipulator's experimental transfer function poles and zeros. The calculated values of transfer function poles and zeros have been compared with the measured values. Moreover, the calculated values of real transfer function zeros bave been used to improve the quality of DSA identification curve fitting process.

\section{STRUCTURAL DYNAMIC MODEL OF A SINGLE LINK FLEXIBLE MANIPULATOR.}

Consider the Pinned-Free Euler-Bernoulli beam shown in Fig. 1. The beam has length 1 , Young's modulus E, area moment of inertia I, mass density per unit volume $\rho$, and crosssectional area $A$. The input is a torque at the pinned end, $M(0, t)$, while the output is the deflection at an arbitrary point $x$ along the beam, $y(x, t)$. It is assumed that both the rigid body and flexible motions are small. The structural vibrational behavior of the beam is described by the following partial differential equation. 


$$
\mathrm{EI} \frac{\partial^{4} \mathrm{y}(\mathrm{x}, \mathrm{t})}{\partial \mathrm{x}^{4}}+\rho \mathrm{A} \frac{\partial^{2} \mathrm{y}(\mathrm{x}, \mathrm{t})}{\partial \mathrm{t}^{2}}=0
$$

The boundary conditions at the pinned end are,

$$
\begin{gathered}
y(0, t)=0 \\
\mathrm{EI} \frac{\partial^{2} \mathrm{y}(0, \mathrm{t})}{\partial \mathrm{x}^{2}}=-\mathrm{M}(0, \mathrm{t})
\end{gathered}
$$

And at the free end are,

$$
\begin{aligned}
& \operatorname{EI} \frac{\partial^{2} y(L, t)}{\partial x^{2}}=0 \\
& \operatorname{EI} \frac{\partial^{3} y(L, t)}{\partial x^{3}}=0
\end{aligned}
$$

The initial conditions are assumed to be zero, i.e., $y(x, 0)=0$ and $y^{\circ}(x, 0)=0$.

Considering the laplace transform of Eqs. (1)-(5) and using the trigonometric and hyperbolic functions, the solution of Eq. (1) can be written as,

$$
y(x, \gamma)=C_{1}(\gamma) \sin \gamma x+C_{2}(\gamma) \cos \gamma x+C_{3}(\gamma) \sinh \gamma x+C_{4}(\gamma) \cosh \gamma x
$$

Where :-

$$
\begin{aligned}
\gamma & =\beta \mathrm{s}^{1 / 2} \quad[1,5] \\
\beta^{4} & =-\rho \mathrm{A} / \mathrm{EI}
\end{aligned}
$$

The coefficients $C_{i}, i=1,2,3$, and 4 in Eq. (6) are found by applying the time transforms of the boundary conditions, expressed by Eqs. (2)-(5). The characteristic roots of Eq. (1) are found and the boundary conditions are applied. This yields the transcendental transfer function from the torque at $x=0$ to the displacement at a general point $x, 0 \leq x \leq L$ as,

$$
\mathrm{G}(\mathrm{x}, \gamma)=\mathrm{y}(\mathrm{x}, \gamma) / \mathrm{M}(0, \gamma)=\mathrm{N}(\mathrm{x}, \gamma) / \mathrm{D}(\mathrm{x}, \gamma)
$$

Where the numerator $\mathrm{N}$ and denominator $\mathrm{D}$ are expressed as $[1,5]$,

$$
\begin{gathered}
\mathrm{N}(\mathrm{x}, \gamma)=\mathrm{a}_{1}(\gamma) \mathrm{b}_{1}(\mathrm{x}, \gamma)+\mathrm{a}_{2}(\gamma) \mathrm{b}_{2}(\mathrm{x}, \gamma)+\mathrm{a}_{3}(\gamma) \mathrm{b}_{3}(\mathrm{x}, \gamma) \\
\mathrm{D}(\gamma)=2 \mathrm{EI} \gamma^{2} \mathrm{a}_{3}(\gamma)
\end{gathered}
$$

Eq. (7), expresses the system transfer function as the ratio the numerator, Eq. (8), and the denominator, Eq. (9). Note that the numerator, which defines the zeros of the transfer function, depends on the sensor position $\mathrm{x}$. 


\subsection{Transfer function poles.}

To justify the product expansion, in Factoring the denominator Eq. (9), has been used by Goodson [6] , Wie [7], and [5]. Since control analysis is usually performed in the s-plane, the s-plane roots are of interest. Each $\gamma$-plane quadruple maps into a complex conjugate pair of imaginary poles in the $s$ - plane:

$$
s_{n}= \pm i(E I / \rho A)^{\frac{1}{2}}\left(d_{n} / 1\right)^{2}
$$

Where $d_{n}$ is the solution of :-

$$
\tan d_{n}=\tanh d_{n} \quad \text { with } d_{n}>0 \text {, real }
$$

Note that, as mentioned before, the poles of the transfer function do not depend on the actuator or sensor locations. In solving Eq. (11) numerically, it is also helpful to note that the higher roots differ by almost exactly $\pi$.

\subsection{Transfer function zeros.}

Analytically factoring the numerator, Eq. (8) using product expansion [6,7], presents greater difficulty, except in the special cases $x=0$ and $x=1$. Note that the numerator which defines the zeros of the transfer function depends on the sensor position $x, 0<x<1$.

\section{A. For sensor at $x=0$}

Since controls analysis is usually performed in the s-plane, the s-plane roots are of interest. Each $\gamma$-plane quadruple map into pure imaginary conjugate zeros. The roots values of $b_{n}$ have been evaluated using the computer program on Matlab Package [5].

$$
\mathrm{s}_{\mathrm{n}}= \pm \mathrm{i}(\mathrm{EI} / \mathrm{\rho A})^{\frac{1}{2}}\left(\mathrm{~b}_{\mathrm{n}} / 1\right)^{2}
$$

Where $b_{n}$ is the solution of:-

$$
1+\cos b_{n} \cosh b_{n}=0 \quad b_{n}>0, \text { real }
$$

\section{B. For Sensor at $x=1$}

The sensor is placed at the tip position of the flexible beam. For $\mathrm{x}=1$, the numerator roots are mapped into pairs of real zeros in the s-plane, with one of each pair in the left half plane and the other in the right half plane. The roots values of $c_{n}$ have been evaluated using the computer program on Matlab Package [5].

$$
s_{n}= \pm 2(E I / \rho A)^{\frac{1}{2}}\left(c_{n} / 1\right)^{2}
$$

Where: $c_{n}$ is the solution of:-

$$
\tan c_{n}+\tanh c_{n}=0, \quad c_{n}>0, \text { real }
$$




\section{For Sensor at General Point.}

For a general point $x, 0<x<1$, the s-plane zeros will be a mixture of real and imaginary pairs. The transfer function zeros determines whether the system is minimum phase or nonminimum phase. For the general case, numerical methods are required to determine the transfer function zeros, based on Matlab Package. For a fixed value of $x$, the first ten transfer function zeros were determined numerical by evaluating the magnitude of the numerator, Eq.
(8), along the following two half lines in the $\gamma$-plane :-

- Case I :

$$
\gamma=\alpha
$$
The ten transfer function zeros were determined numerically by evaluating the magnitude of
the numerator Eq. (8) where :-

$$
\begin{gathered}
N(x, \alpha)=(1+\cos \alpha 1 \cosh \alpha 1)(\sin \alpha x+\sinh \alpha x)+(\sin \alpha 1 \sinh \alpha 1)(\sin \alpha x-\sinh \alpha x)+ \\
(\cos x \sinh x-\sin \alpha 1 \cosh \alpha 1)(\cos \alpha x-\cosh \alpha x)
\end{gathered}
$$

These roots map into a complex conjugate pair of pure imaginary s-plane zeros:-

$$
\mathrm{s}= \pm \sqrt{\mathrm{EI} / \rho \mathrm{A}} \alpha^{2}
$$

For a fixed value of $x$, the first ten transfer function zeros were determined numerically by evaluating the magnitude of the numerator Eq. (17), along the half line in the $\gamma$-plane Eq. (16). The roots in the $\gamma$-plane as $\mathrm{x}$ is varied from 0 to 1 , are evaluated in [5].

- Case II :

$$
\gamma=\alpha(1+i)
$$

These roots map into a pair of real s-plane zeros, with one of each pair in the left half plane and the other in the right half plane.

$$
\mathrm{s}= \pm 2 \sqrt{\mathrm{EI} / \rho \mathrm{A}} \alpha^{2}
$$

For a fixed value of $\mathrm{x}$, the first ten transfer function zeros were determined numerically by evaluating the magnitude of the numerator Eq. (8), along the half line in the $\gamma$-plane Eq. (19). The roots in the $\gamma$-plane as $\mathrm{x}$ is varied from 0 to 1 , are evaluated in [5].

\section{CASE STUDY}

For the sake of comparison between the computed values of transfer function poles and zeros and the experimentally identified ones a case study has been investigated. The schematic of the flexible robot arm used in this investigation is shown in Fig. 2. The one-link flexible robot arm rotates in the horizontal plane, is constructed from a single continuous aluminum beam and the only control available is the motor located at the single rotating joint. 
The flexible beam to be modeled has dimensions and material properties listed in Table 1. Synthesized frequency response curves are obtained based on the computed poles and zeros. These poles and zeros have been evaluated using the computer program on Matlab Package.

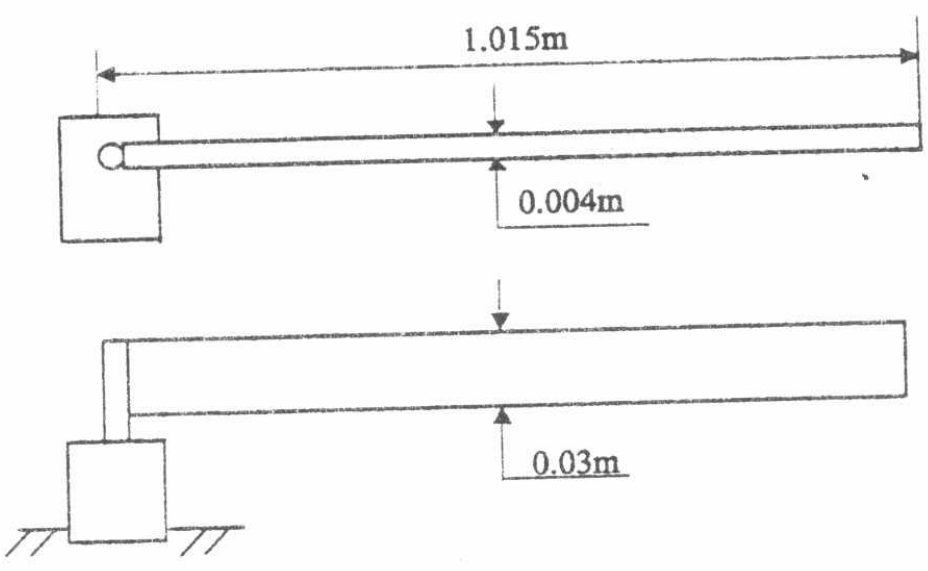

Fig. 2 The flexible manipulator under investigation

Table 1 Dimensions and material properties of flexible beam

\begin{tabular}{|l|l|}
\hline Material & Aluminum \\
Modulus of elasticity $\mathrm{E}\left(\mathrm{N} / \mathrm{m}^{2}\right)$ & $72.38 \times 10^{9}$ \\
Volume density $\mathrm{p}\left(\mathrm{kg} / \mathrm{m}^{3}\right)$ & 2767.54 \\
Length $\mathrm{l}(\mathrm{m})$ & 1.015 \\
Height $\mathrm{h}(\mathrm{m})$ & $3.0 \times 10^{-2}$ \\
Width $\mathrm{t}(\mathrm{m})$ & $4.0 \times 10^{-3}$ \\
Total mass $(\mathrm{kg})$ & $2.6 \times 10^{-1}$ \\
Cross section area $\left(\mathrm{m}^{2}\right)$ & $1.2 \times 10^{-4}$ \\
Moment of inertia $\left(\mathrm{m}^{4}\right)$ & $1.6 \times 10^{-10}$ \\
\hline
\end{tabular}

\section{EXPERIMENTAL INVESTIGATION}

The experimental setup is schematically represented in Fig. 3. It consists of a flexible link shown in Fig. 2. The link is made of aluminum alloy. Its cross section is designed to provide considerable flexibility in the lateral direction while maintaining sufficient rigidity in the vertical direction. The material properties of the link are given in Table 1 . With a DC permanent magnet motor unit installed on the hub. 
The experimental setup included also a Modified push-pull power amplifier to measure the current passing through the armature windings of the DC motor, the motor current is sensed by a sensed resistor that is small compared with motor resistance. Two measurements are available, the current passing through the armature winding of the DC motor, and an acceleration sensor located along the beam, to provide the output charge proportional to the acceleration of the vibrated beam. Charge amplifier with active integration networks are built in for conversion of vibration acceleration to displacement signals, and HP 3562A Dynamic Signal Analyzer used to measure the frequency response with swèpt sine mode.

The prediction of the previous synthesized frequency response section have been checked and verified, using the HP 3562A Dynamic Signal Analyzer. Based on measuring the open-loop frequency response transfer function between the applied torque from the dc permanent magnet motor, and the position output response from charge amplifier. Apply harmonic swept sine waveform from the Dynamic Signal Analyzer source, with source limit 1 volt, and frequency band $11.5 \mathrm{~Hz}-720 \mathrm{~Hz}$, to the power amplifier $\mathrm{V}_{\mathrm{i}}$. The amplified voltage signal $\mathrm{V}_{\mathrm{a}}$, drives the $\mathrm{DC}$ permanent magnet motor and produce an output current proportional to the command voltage $\mathrm{V}_{\mathrm{i}}$, which is proportional to the generated motor torque.

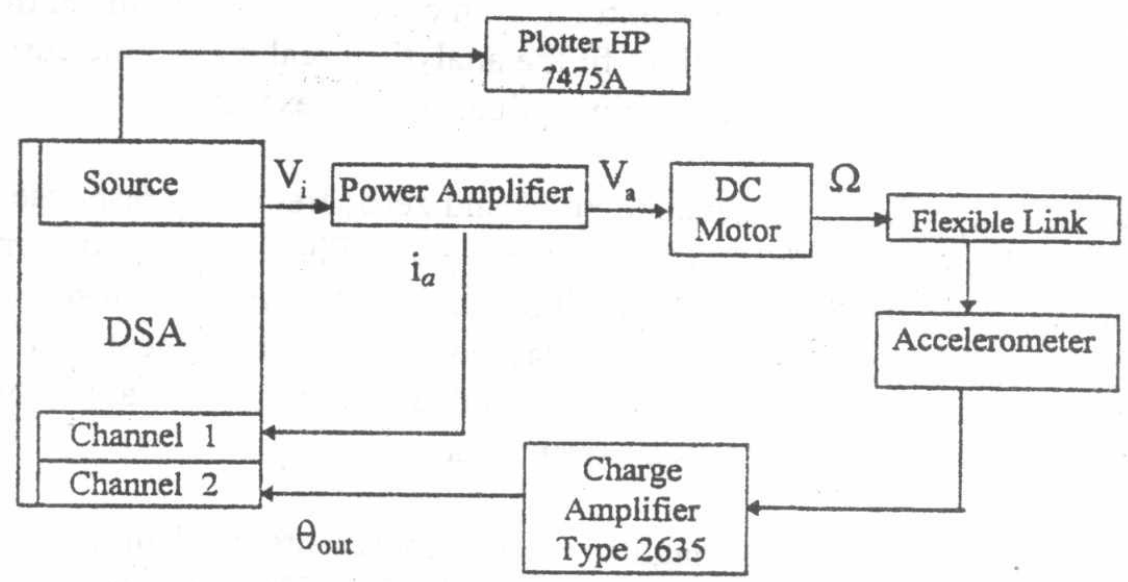

Fig. 3. Schematic diagram of experimental setup

The motor current sensed by a shunt resistance, is fed back to channel 1 of the analyzer. This is made with the objective to measure the harmonic current passing throughout the armature windings of the dc motor. This current is proportional with the torque generated, which actuate the flexible link at the revolute pinned joint. An accelerometer located at different position along the flexible beam, measures the acceleration of the beam, at $x=0.8$, and 1 . Charge amplifier with active integration networks are built in for conversion of vibrational acceleration to displacement signals. This displacement signal is the output response of the flexible beam, and fed back to channel 2 of the analyzer, to measure the frequency response of the flexible beam model. 


\section{RESULTS AND DISCUSSION.}

The measurements show both the open loop frequency response of the flexible beam model as a bode plot, and the coherence. The coherence is a functional of frequency, each frequency point in the coherence function is a real value between 0.0 , and 1.0. A value of 1.0 implies that noise does not contaminate the measurement at that frequency. Using the powerful tool allowed in the analyzer, to get the coefficients for a function that best fits the ineasured frequency response data, to closely approximate the frequency response obtained from the measurement. The poles and zeros model is developed by calculating a weighted least-squares fit of the frequency response data. With curve fitting we will start with frequency response trace magnitude curve, or phase curve, whatever, to find a linear model that correspond to the trace.

Generally, these arms have highly vibratory poles and low damping factors, and the measured frequency response have a wide band of frequencies. Then we have the option to enter and "fix" known system transfer function poles and zeros, the known poles and zeros can be obtained from curve fitting a narrow region around a resonance. This is done by decreasing the entire frequency response trace displayed on the analyzer is used as fit region. Then the poles and the imaginary zeros extract from the curve fitting with narrow region are fixed. Except that the real zeros, we can not extract from curve fitting with the narrow band region, and we have been forced the curve fit to fit the analytical real zeros. The curve fitter assumes they are correct and includes them as starting values in the next fit.

Fig. 4 shows the synthesized calculated poles and zeros frequency response bode plot of the single link flexible manipulator for $\mathrm{x}=0.8$, and $\mathrm{x}=1.0$. Fig. 5 shows experimental frequency response bode plot of the single link flexible manipulator which represented as a solid line, and the corresponding curve fit shown as a dashed line for sensor located at $\mathrm{x}=0.8$, and 1.0. A comparison table between the experimental and analytical results, are shown in Table 2 . In view of Fig. 4, Fig. 5, and Table 2 it is apparent that :

The real part for both poles and imaginary zeros, represents the damping of the flexible link theoretically are assumed to have a negligible value. This real part of both poles and imaginary zeros have been determined experimentally. The imaginary part for both poles and zeros experimentally shows nearly the same analytically values specially at low frequency range. Except that their exist a slight difference at the high frequencies. It is clear that from theoretical analysis the imaginary zeros have a phase lead 180 degree, but experimentally their exist imaginary zeros hiave a very high damping factor, forced the phase to be lag -180 degree, resulting in increasing the nonminimum phase.

Although in theoretical analysis the rigid body pinned mode are represents by a two poles at the origin in the s-plane, but from experimental results we have been estimated. The calculated real zeros are used as a fixed values in the curve fit table for adjustment the curve fitting of the measurement frequency response. 
Proceedir:'s of itie $7^{\text {th }}$ ASAT Conf. 13-15 May 1997

\begin{tabular}{|l|l|}
\hline SM-12 & 335 \\
\hline
\end{tabular}
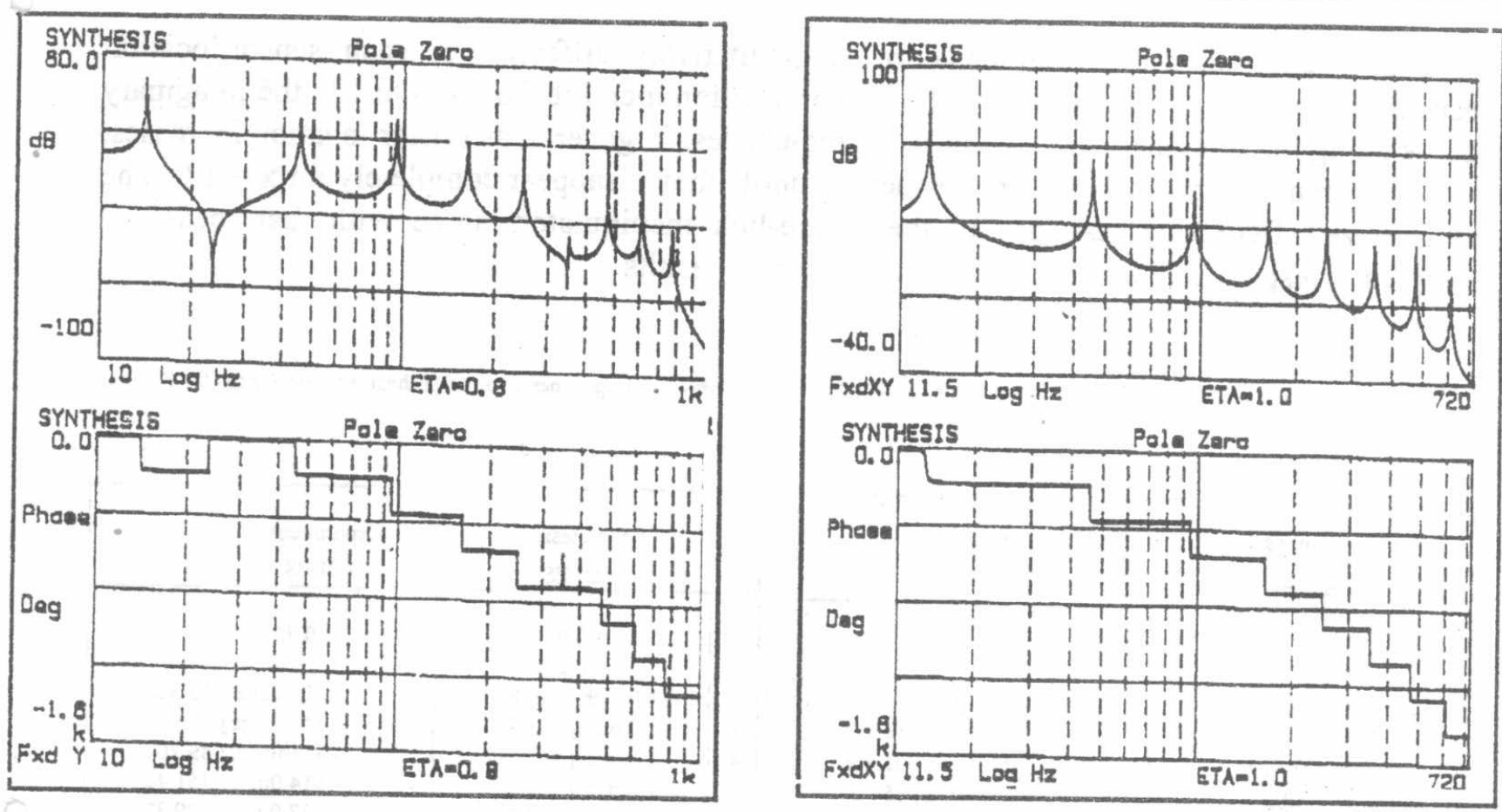

$$
\mathrm{x}=0.8
$$

$$
\mathrm{x}=1.0
$$

Fig. 4 Synthesized torque to displacement transfer function
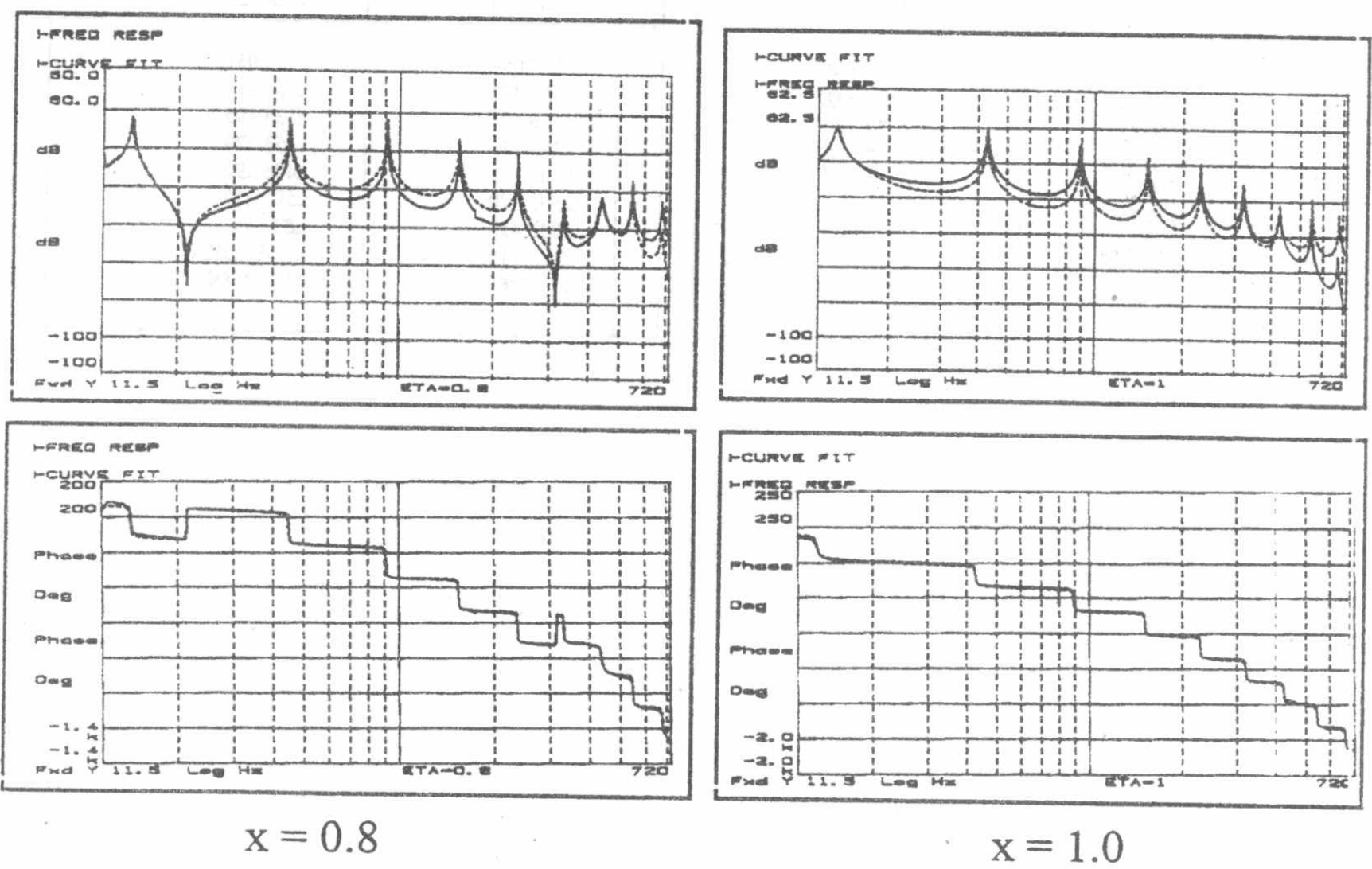

$\mathrm{X}=0.8$

Fig. 5 Measured frequency response curves 
It is apparent that the system behavior is qualitatively different for each sensor location especially at low frequencies. As the sensor location increase from 0.8 to 1.0 , the imaginary zeros along the imaginary axis increase in frequencies. In general, as sensor location increases, the imaginary zeros increase in frequency until they disappear completely for $x=1.0$. The synthesized frequency response of the single-link manipulator, agree with that measured frequency response obtained from the experimental results.

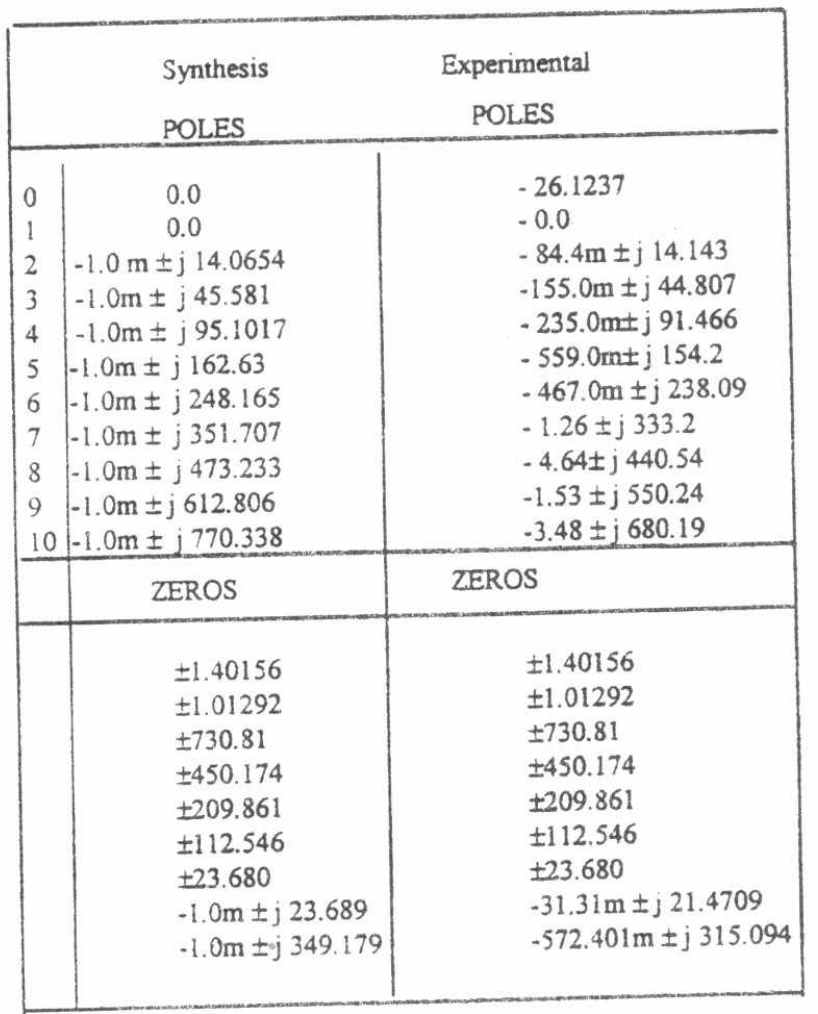

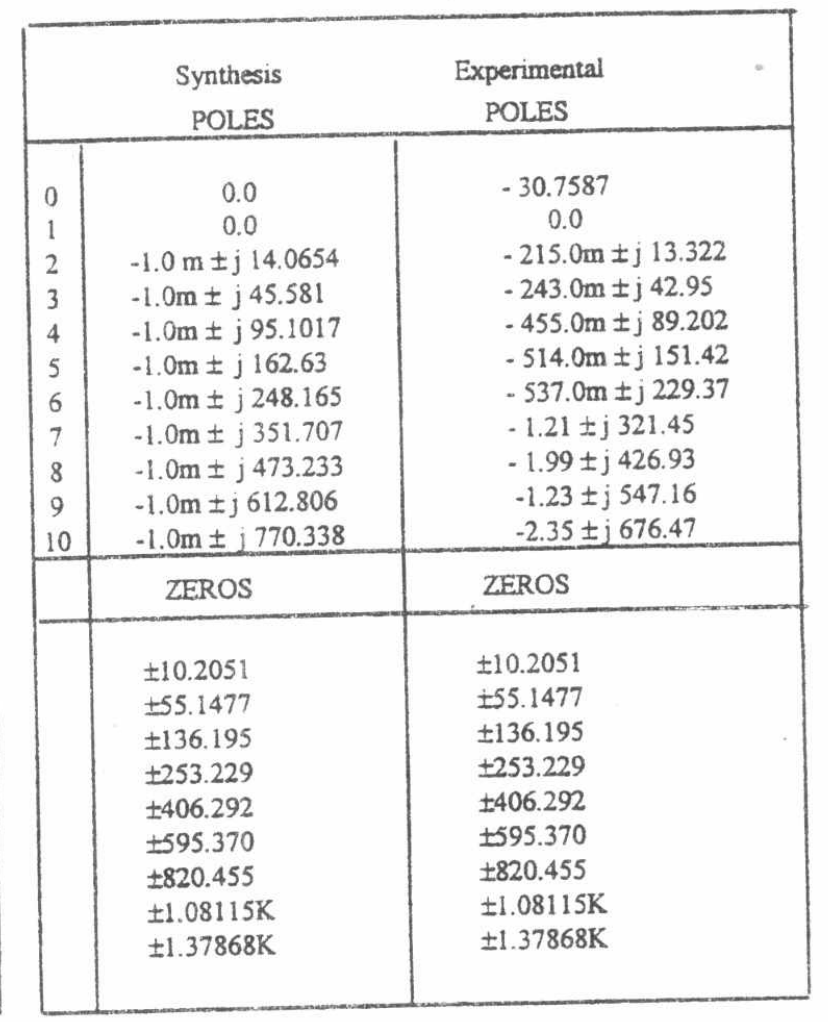




\section{CONCLUSION}

Position control of flexible mechanical systems is an important topic. Its application range from control of motor-driven-actuators in computer peripherals, to robotics manipulators and large scale active space structures. Much excellent work has been done in this area, especially those related to instability caused by the noncollocation of senșors and actuators. It is well known that the control system will be stable when there exists alternating poles and zeros. Mechanical engineers have good physical understanding of the poles. Complex conjugate poles are the resonant frequencies of the flexible structure. The purpose of this paper is to discuss the modeling of the flexible beam, physical significance of the zeros, and experimental study. Its shown that the zeros are related to the energy propagation characteristics of a flexible system specifically, for the case of uniform cross-section elastic beams, the complex conjugate zeros are shown to be the resonant frequencies of the constrained substructure and are related to propagation of energy. The real zeros, which cause the control system to be nonminimum phase, are related to nonpropagation of energy. The example treated here has the generic properties of more complex structural control problems. Therefore the analysis, and experiments presented here lead to the following general
observations:

1. Accurate dynamic modeling of the structure is of critical importance is noncollocated structural control design, small inaccuracies in the model can lead to qualitatively different system characteristics, resulting in deficient and possibly unstable designs. In particular, small variations in sensor locations can result in interchanging the ordering of poles and zeros, producing phase errors of up to -360 degrees. A phase error of this size virtually guarantees closed loop instability.

2. Noncollocated structural control systems are always nonminimum phase above some finite frequency. The frequency at which the transfer function becomes nonminimum phase decreases as the sensor/actuator distance increases. A conventional modal model, that is perfectly adequate for collocated control design, may therefore be completely unsuitable for noncollocated control design.

\section{REFERENCES}

1. V. A. Spector, H. Flashner, "Modeling and Design Implications of Noncollocated Control in Flexible Systems" Transaction of the ASME, Vol. 112, June 1990, pp. 186-193.

2. W. T. Qian and C. C. H. Ma., "A New Controller design for controlling a Flexible OneLink Manipulator" IEEE Transactions on Automatic Control, Vol. 37, No. 1, January 1992, pp. 132-137.

3. D. K. Miu., "Physical Interpretation of Transfer Function Zeros for Simple Control Systems With Mechanical Flexibility" Journal of Dynamic Systems, Measurement, and Control, Vol. 113, September 1991, pp. 419-424. 
4. C. H. Menq, and J. Z. Xia, "Experiments on the Tracking Control of a flexible One-Link Manipulator" Transactions of the ASME, Vol. 115, June 1993, pp. 306-308.

5. A. E. Shawky, "Motion Control of Flexible Manipulators," Master of Science In Electrical Engineering, M.T.C. Cairo, Egypt, February, 1987.

6. R. E. Goodson, "Distributed System Simulation Using Infinite Product Expansions," Simulation, Dec. 1970, pp. 255-263.

7. B. Wie, "On the Modeling and control of Flexible Space Structure," SUDAAR 525, Stanford University Guidance and Control Laboratory, June 1981.

8. R. Cannon, and E. Schmitz,"Initial Experiments on the point control of a flexible One-link robot", the Int., J. of robotic Research, Vol. 3, No. 3, PP 62-75,1984.

9. A. S. Abdel Mohsen E.T. Abou El Dahab. "On the Interaction between the position and control, and the Dynamics of a Flexible Robot Manipulator" The 11th ISPE/IEE/IFAC international conference on CAD/CAM and Factories of Future '95, 28-30 August 1995, Pereira, Colombia, S. America.

\section{NOMENCLATURE}

\begin{tabular}{|c|c|c|}
\hline A & : & Cross sectional area $\left(\mathrm{m}^{2}\right)$ \\
\hline $\mathbf{a}(\gamma)$ & : & Position-independent terms \\
\hline$b_{n}$ & : & Numerator roots at $\mathrm{x}=0$ \\
\hline$c_{n}$ & : & Numerator roots at $x=1$ \\
\hline $\mathrm{D}(\mathbf{x}, \gamma)$ & : & Denominator of the transcendental transfer function \\
\hline$d_{n}$ & : & Denominator roots \\
\hline E & : & Modulus of elasticity $\left(\mathrm{N} / \mathrm{m}^{2}\right)$ \\
\hline $\mathrm{f}(\mathrm{x}, \gamma)$ & : & Position-dependent terms \\
\hline $\mathrm{G}(\mathbf{x}, \gamma)$ & : & Transcendental transfer function \\
\hline 1 & : & The beam length $(\mathrm{m})$ \\
\hline $\mathbf{M}(0, \mathrm{~s})$ & : & Applied torque at the pinned end (N.m) \\
\hline $\mathbf{N}(\mathbf{x}, \gamma)$ & : & Numerator of the transcendental transfer function \\
\hline I & : & Moment of inertia $\left(\mathrm{m}^{4}\right)$ \\
\hline$y(x, t)$ & : & Deflection at an arbitrary point $\mathrm{x}(\mathrm{m})$ \\
\hline$\rho$ & : & Mass density per unit volume $\left(\mathrm{Kg} / \mathrm{m}^{3}\right)$ \\
\hline$\gamma-\mathrm{s}$ & : & Transformation plane \\
\hline$\alpha$ & : & Wave number parameter \\
\hline$\eta=x / 1$ & : & Normalized separation \\
\hline $\mathrm{DC}$ & : & Direct Current \\
\hline DSA & : & Dynamic Signal Analyzer \\
\hline PDE & : & Partial Differential Equation \\
\hline
\end{tabular}

